\title{
Use of $\alpha$-resolvable designs in the construction of two-factor experiments of split-plot type
}

\author{
Kazuhiro Ozawa ${ }^{1}$, Shinji Kuriki ${ }^{2}$, Stanisław Mejza ${ }^{3}$ \\ ${ }^{1}$ Department of Nursing, Gifu College of Nursing, Hashima, Gifu, 501-6295, Japan, \\ e-mail: ozawa@gifu-cn.ac.jp \\ ${ }^{2}$ Department of Mathematical Sciences, Graduate School of Engineering, Osaka \\ Prefecture University, Naka-ku, Sakai, Osaka, 599-8531, Japan, \\ e-mail: kuriki@ms.osakafu-u.ac.jp \\ ${ }^{3}$ Department of Mathematical and Statistical Methods, Poznań University of Life \\ Sciences, Wojska Polskiego 28, 60-637 Poznań, Poland, e-mail: smejza@up.poznan.pl
}

\section{SUMMARY}

We consider an incomplete split-plot design (ISPD) with two factors generated by the semi-Kronecker product of two $\alpha$-resolvable designs. We use an $\alpha$-resolvable design for the whole plot treatments and an affine $\alpha$-resolvable design for the subplot treatments. We characterize the ISPDs with respect to the general balance property, and we give the stratum efficiency factors for the ISPDs.

Key words: $\alpha$-resolvable designs; Affine $\alpha$-resolvable designs; General balance property; Incomplete split-plot designs; Stratum efficiency factors.

\section{Introduction}

We consider a two-factor experiment of split-plot type. The first factor $A$ has $v_{1}$ levels $A_{1}, A_{2}, \ldots, A_{v_{1}}$, and the second factor $B$ has $v_{2}$ levels $B_{1}, B_{2}, \ldots, B_{v_{2}}$. In a split-plot design, each of $b$ blocks is divided into $k_{1}$ whole plots, and each whole plot is divided into $k_{2}$ subplots. The levels of factors $A$ and $B$ are applied to the whole plots (called whole plot treatments) and the subplots (called subplot treatments), respectively. We consider an incomplete split-plot design (ISPD) such that $k_{1}<v_{1}$ or $k_{2}<v_{2}$.

ISPDs are used in biological, agricultural and environmental sciences, and the analysis of data and the constructions of the ISPDs have been studied by Bhargava and Shah (1975), Rees (1969), Robinson (1967a, 1967b), Mejza and Mejza (1984, 1996), Mejza (1987), and others. In Mejza (1987) 
and Mejza and Mejza (1996), a mixed linear model with fixed treatment effects and random block, whole plot and subplot effects was given. The $h$ th factorial treatment combination effect $\tau_{h}$ is defined by

$$
\tau_{h}=\mu+\alpha_{i}+\gamma_{j}+(\alpha \gamma)_{i j}
$$

for $h=(i-1) v_{2}+j, i=1,2, \ldots, v_{1}$ and $j=1,2, \ldots, v_{2}$, where $\mu$ is the overall mean, $\alpha_{i}$ denotes the main effect of the level $A_{i}$ of the factor $A$, $\gamma_{j}$ denotes the main effect of the level $B_{j}$ of the factor $B$ and $(\alpha \gamma)_{i j}$ denotes the interaction effect of $A_{i}$ and $B_{j}$. Here $\Sigma_{i=1}^{v_{1}} \alpha_{i}=0, \Sigma_{j=1}^{v_{2}} \gamma_{j}=0$, $\Sigma_{i=1}^{v_{1}}(\alpha \gamma)_{i j}=0$ for $j=1,2, \ldots, v_{2}$ and $\Sigma_{j=1}^{v_{2}}(\alpha \gamma)_{i j}=0$ for $i=1,2, \ldots, v_{1}$. The mixed linear model results from a three-step randomization, i.e., the randomization of blocks, the randomization of whole plots within each block and the randomization of subplots within each whole plot. This kind of randomization leads us to the experiments with orthogonal block structure as defined by Nelder (1965a, 1965b), and the multistratum analysis proposed by Nelder $(1965 \mathrm{a}, 1965 \mathrm{~b})$ and Houtman and Speed (1983) can be applied to the analysis of the experiments. In the case of the ISPD, we have three strata, apart from the zero stratum connected with the overall mean only: (I) the inter-block stratum, (II) the inter-whole plot stratum and (III) the intra-unit stratum. The statistical properties of the ISPD are strongly related to the eigenvalues and the eigenvectors of the stratum information matrices for the treatment combinations. Here, we assume that every treatment combination $A_{i} B_{j}\left(i=1,2, \ldots, v_{1}, j=1,2, \ldots, v_{2}\right)$ occurs in precisely $r$ blocks and the treatment combinations are arranged in the lexicographic order. The stratum information matrices are

$$
\begin{aligned}
& \mathbf{A}_{1}=\frac{1}{k_{1} k_{2}} \mathbf{N}_{1} \mathbf{N}_{1}^{\prime}-\frac{r}{v} \mathbf{J}_{v}, \quad \mathbf{A}_{2}=\frac{1}{k_{2}} \mathbf{N}_{2} \mathbf{N}_{2}^{\prime}-\frac{1}{k_{1} k_{2}} \mathbf{N}_{1} \mathbf{N}_{1}^{\prime} \\
& \text { and } \mathbf{A}_{3}=r \mathbf{I}_{v}-\frac{1}{k_{2}} \mathbf{N}_{2} \mathbf{N}_{2}^{\prime},
\end{aligned}
$$

where $v=v_{1} v_{2}, \mathbf{N}_{1}$ and $\mathbf{N}_{2}$ are the incidence matrices for treatment combinations vs. blocks and treatment combinations vs. whole plots, respectively, $\mathbf{I}_{v}$ is the identity matrix of order $v$ and $\mathbf{J}_{v}$ is a $v \times v$ matrix whose elements are all unity. The eigenvalues of a matrix $\mathbf{A}_{f}^{*}=r^{-1} \mathbf{A}_{f}$ can be identified as the stratum efficiency factors of the design for $f=1,2,3$ (see Houtman and Speed, 1983). Moreover, the eigenvectors define contrasts of treatment effects, which are called the basic contrasts (see Pearce, Caliński and Marshall, 1974). 
Mejza (1987) and Mejza and Mejza (1996) considered the constructions of ISPDs by the Kronecker product of the incidence matrices of two designs. Ozawa et al. (2004), Ozawa and Kuriki (2006) and Kuriki and Nakajima (2007) considered the constructions of ISPDs by a modified Kronecker product (called the semi-Kronecker product) of the incidence matrices of two resolvable designs. In this paper, we construct a new ISPD by the semiKronecker product of the incidence matrices of an $\alpha$-resolvable design for the whole plot treatments and an affine $\alpha$-resolvable design for the subplot treatments, and these new ISPDs also include those obtained by Ozawa et al. (2004), Ozawa and Kuriki (2006) and Kuriki and Nakajima (2007). We characterize the ISPDs with respect to the general balance property and we give the stratum efficiency factors for the ISPDs. These ISPDs have smaller numbers of blocks than those designs constructed by the Kronecker product of two designs, and they would be useful in many practical situations.

\section{Method of construction of ISPDs}

Firstly, we need the semi-Kronecker product of two matrices in order to construct ISPDs (see Mejza, Kuriki and Mejza, 2001). Suppose that two matrices $\mathbf{E}$ and $\mathbf{F}$ are divided into the same number $t$ of submatrices as follows:

$$
\mathbf{E}=\left(\mathbf{E}_{1}: \mathbf{E}_{2}: \cdots: \mathbf{E}_{t}\right) \quad \text { and } \quad \mathbf{F}=\left(\mathbf{F}_{1}: \mathbf{F}_{2}: \cdots: \mathbf{F}_{t}\right) .
$$

Then, the semi-Kronecker product $\mathbf{E} \tilde{\otimes} \mathbf{F}$ is given by

$$
\mathbf{E} \tilde{\otimes} \mathbf{F}=\left(\mathbf{E}_{1} \otimes \mathbf{F}_{1}: \mathbf{E}_{2} \otimes \mathbf{F}_{2}: \cdots: \mathbf{E}_{t} \otimes \mathbf{F}_{t}\right),
$$

where $\otimes$ denotes the usual Kronecker product. This type of Kronecker product was first considered by Khatri and Rao (1968).

Next, we need an $\alpha$-resolvable design and an affine $\alpha$-resolvable design. A design with $v$ treatments, $r$ replications of each treatment and $k$ plots within each block is denoted by $\mathrm{D}(v, r, k)$. If the collection of blocks of a $\mathrm{D}(v, r, k)$ can be partitioned into classes (called resolution classes) such that every treatment occurs precisely $\alpha$ times in every class, the design is said to be $\alpha$-resolvable (resolvable for brevity, if $\alpha=1$ ) and it is denoted by $\alpha-\mathrm{RD}(v, r, k)$. The parameters of an $\alpha-\operatorname{RD}(v, r, k)$ satisfy the following conditions:

$$
\alpha v=k \beta, \quad b=t \beta \quad \text { and } \quad r=\alpha t,
$$


where $\beta$ is the number of blocks within each resolution class, $b$ is the total number of blocks and $t$ is the number of resolution classes. Moreover, an $\alpha$-resolvable design is called an affine $\alpha$-resolvable design if the number of common treatments appearing in two distinct blocks in the same resolution class is $q_{1}$, and if the number of common treatments appearing in two distinct blocks belonging to different resolution classes is $q_{2}$. The parameters of an affine $\alpha-\operatorname{RD}(v, r, k)$ satisfy the additional following conditions:

$$
q_{1}=\frac{\alpha-1}{\beta-1} k, \quad q_{2}=\frac{k^{2}}{v}, \quad \beta>1 \quad \text { and } \quad k>q_{1} .
$$

Many authors have given constructions of $\alpha$-resolvable and affine $\alpha$-resolvable balanced and partially balanced incomplete block designs (see, for example, Kageyama, 1973, Bailey, Monod and Morgan, 1995, Caliński and Kageyama, 2003, Kadowaki and Kageyama, 2009). By taking some resolution classes of these designs, we can obtain $\alpha$-resolvable and affine $\alpha$-resolvable designs considered above. In particular, an affine $\alpha$-resolvable design with $\alpha=1$, $q_{1}=0$ and $q_{2}=1$ is called a square lattice design.

We construct an ISPD from an $\alpha$-resolvable design and an affine $\alpha$ resolvable design. Let

$$
\mathbf{N}_{A}=\left(\mathbf{N}_{A 1}: \mathbf{N}_{A 2}: \cdots: \mathbf{N}_{A t}\right) \text { and } \mathbf{N}_{B}=\left(\mathbf{N}_{B 1}: \mathbf{N}_{B 2}: \cdots: \mathbf{N}_{B t}\right)
$$

be the incidence matrices of an $\alpha_{1}-\operatorname{RD}\left(v_{1}, r_{1}, k_{1}\right)$ with $\beta_{1}=\alpha_{1} v_{1} / k_{1}$ and $b_{1}=t \beta_{1}$, and an affine $\alpha_{2}-\operatorname{RD}\left(v_{2}, r_{2}, k_{2}\right)$ with $\beta_{2}=\alpha_{2} v_{2} / k_{2}$ and $b_{2}=t \beta_{2}$, respectively, where the designs have the same number $t$ of resolution classes, and $\mathbf{N}_{A i}$ and $\mathbf{N}_{B i}$ correspond to the $i$ th resolution class. By the definition of the affine $\alpha$-resolvable design,

$$
\mathbf{N}_{B i}^{\prime} \mathbf{N}_{B i}=\left(k_{2}-q_{21}\right) \mathbf{I}_{\beta_{2}}+q_{21} \mathbf{J}_{\beta_{2}} \quad \text { and } \quad \mathbf{N}_{B i}^{\prime} \mathbf{N}_{B j}=q_{22} \mathbf{J}_{\beta_{2}}
$$

hold for $i, j=1,2, \ldots, t, i \neq j$, where $q_{21}=k_{2}\left(\alpha_{2}-1\right) /\left(\beta_{2}-1\right)$ and $q_{22}=k_{2}^{2} / v_{2}$. Now we construct an ISPD, say $\tilde{\mathcal{D}}$, such that its incidence matrix $\mathbf{N}_{1}$ is obtained by the semi-Kronecker product of $\mathbf{N}_{A}$ and $\mathbf{N}_{B}$, i.e.,

$$
\mathbf{N}_{1}=\mathbf{N}_{A} \tilde{\otimes} \mathbf{N}_{B}=\left(\mathbf{N}_{A 1} \otimes \mathbf{N}_{B 1}: \mathbf{N}_{A 2} \otimes \mathbf{N}_{B 2}: \cdots: \mathbf{N}_{A t} \otimes \mathbf{N}_{B t}\right) \text {. }
$$

Here, we use the $\alpha$-resolvable design for whole plot treatments and the affine $\alpha$-resolvable design for subplot treatments, respectively, and we arrange the same treatments of the affine $\alpha$-resolvable design on the subplots in each whole plot within each block. The ISPD $\tilde{\mathcal{D}}$ has $v_{1}$ whole plot treatments, $v_{2}$ subplot treatments and $t \beta_{1} \beta_{2}$ blocks with $k_{1}$ whole plots and $k_{2}$ subplots in 
each whole plot. For $\tilde{\mathcal{D}}$, we can express $\mathbf{N}_{2}$ as

$$
\mathbf{N}_{2}=\left(\mathbf{1}_{\alpha_{1}}^{\prime} \otimes \mathbf{I}_{v_{1}} \otimes \mathbf{N}_{B 1}: \mathbf{1}_{\alpha_{1}}^{\prime} \otimes \mathbf{I}_{v_{1}} \otimes \mathbf{N}_{B 2}: \cdots: \mathbf{1}_{\alpha_{1}}^{\prime} \otimes \mathbf{I}_{v_{1}} \otimes \mathbf{N}_{B t}\right),
$$

arranging the whole plots in a suitable order, where $\mathbf{1}_{v}$ is a $v \times 1$ vector whose elements are all unity. Therefore, from (3) and (4), we have

$$
\begin{aligned}
& \mathbf{N}_{1} \mathbf{N}_{1}^{\prime}=\sum_{i=1}^{t}\left(\mathbf{N}_{A i} \mathbf{N}_{A i}^{\prime} \otimes \mathbf{N}_{B i} \mathbf{N}_{B i}^{\prime}\right) \text { and } \\
& \mathbf{N}_{2} \mathbf{N}_{2}^{\prime}=\sum_{i=1}^{t}\left(\alpha_{1} \mathbf{I}_{v_{1}} \otimes \mathbf{N}_{B i} \mathbf{N}_{B i}^{\prime}\right)=\alpha_{1} \mathbf{I}_{v_{1}} \otimes \mathbf{N}_{B} \mathbf{N}_{B}^{\prime} .
\end{aligned}
$$

Note that $\tilde{\mathcal{D}}$ is also an $\alpha$-resolvable design with $\alpha=\alpha_{1} \alpha_{2}$ when the treatment combinations are regarded as the usual treatments.

Example 1. We consider a $2-\mathrm{RD}(6,4,4)$ and an affine $2-\mathrm{RD}(9,4,6)$ with $\beta_{1}=\beta_{2}=3, b_{1}=b_{2}=6, t=2, q_{21}=3, q_{22}=4$ and the incidence matrices $\mathbf{N}_{A}=\left(\mathbf{N}_{A 1}: \mathbf{N}_{A 2}\right)$ and $\mathbf{N}_{B}=\left(\mathbf{N}_{B 1}: \mathbf{N}_{B 2}\right)$, where

$$
\begin{aligned}
& \mathbf{N}_{A 1}=\left(\begin{array}{lll}
1 & 1 & 0 \\
1 & 1 & 0 \\
1 & 0 & 1 \\
1 & 0 & 1 \\
0 & 1 & 1 \\
0 & 1 & 1
\end{array}\right), \mathbf{N}_{A 2}=\left(\begin{array}{lll}
1 & 0 & 1 \\
1 & 1 & 0 \\
0 & 1 & 1 \\
0 & 1 & 1 \\
1 & 0 & 1 \\
1 & 1 & 0
\end{array}\right), \\
& \mathbf{N}_{B 1}=\left(\begin{array}{lll}
1 & 0 & 1 \\
1 & 0 & 1 \\
1 & 0 & 1 \\
1 & 1 & 0 \\
1 & 1 & 0 \\
1 & 1 & 0 \\
0 & 1 & 1 \\
0 & 1 & 1 \\
0 & 1 & 1
\end{array}\right), \mathbf{N}_{B 2}=\left(\begin{array}{lll}
1 & 0 & 1 \\
1 & 1 & 0 \\
0 & 1 & 1 \\
1 & 0 & 1 \\
1 & 1 & 0 \\
0 & 1 & 1 \\
1 & 0 & 1 \\
1 & 1 & 0 \\
0 & 1 & 1
\end{array}\right) .
\end{aligned}
$$

The ISPD $\tilde{\mathcal{D}}$ constructed by the semi-Kronecker product has 18 blocks with 4 whole plots and 6 subplots in each whole plot. The ISPD can be expressed by the following way:

$$
\begin{aligned}
& \left\{A_{1}, A_{2}, A_{3}, A_{4} \mid B_{1}, B_{2}, B_{3}, B_{4}, B_{5}, B_{6}\right\},\left\{A_{1}, A_{2}, A_{3}, A_{4} \mid B_{4}, B_{5}, B_{6}, B_{7}, B_{8}, B_{9}\right\}, \\
& \left\{A_{1}, A_{2}, A_{3}, A_{4} \mid B_{1}, B_{2}, B_{3}, B_{7}, B_{8}, B_{9}\right\},\left\{A_{1}, A_{2}, A_{5}, A_{6} \mid B_{1}, B_{2}, B_{3}, B_{4}, B_{5}, B_{6}\right\}, \\
& \left\{A_{1}, A_{2}, A_{5}, A_{6} \mid B_{4}, B_{5}, B_{6}, B_{7}, B_{8}, B_{9}\right\},\left\{A_{1}, A_{2}, A_{5}, A_{6} \mid B_{1}, B_{2}, B_{3}, B_{7}, B_{8}, B_{9}\right\},
\end{aligned}
$$




$$
\begin{aligned}
& \left\{A_{3}, A_{4}, A_{5}, A_{6} \mid B_{1}, B_{2}, B_{3}, B_{4}, B_{5}, B_{6}\right\},\left\{A_{3}, A_{4}, A_{5}, A_{6} \mid B_{4}, B_{5}, B_{6}, B_{7}, B_{8}, B_{9}\right\}, \\
& \left\{A_{3}, A_{4}, A_{5}, A_{6} \mid B_{1}, B_{2}, B_{3}, B_{7}, B_{8}, B_{9}\right\},\left\{A_{1}, A_{2}, A_{5}, A_{6} \mid B_{1}, B_{2}, B_{4}, B_{5}, B_{7}, B_{8}\right\}, \\
& \left\{A_{1}, A_{2}, A_{5}, A_{6} \mid B_{2}, B_{3}, B_{5}, B_{6}, B_{8}, B_{9}\right\},\left\{A_{1}, A_{2}, A_{5}, A_{6} \mid B_{1}, B_{3}, B_{4}, B_{6}, B_{7}, B_{9}\right\}, \\
& \left\{A_{2}, A_{3}, A_{4}, A_{6} \mid B_{1}, B_{2}, B_{4}, B_{5}, B_{7}, B_{8}\right\},\left\{A_{2}, A_{3}, A_{4}, A_{6} \mid B_{2}, B_{3}, B_{5}, B_{6}, B_{8}, B_{9}\right\}, \\
& \left\{A_{2}, A_{3}, A_{4}, A_{6} \mid B_{1}, B_{3}, B_{4}, B_{6}, B_{7}, B_{9}\right\},\left\{A_{1}, A_{3}, A_{4}, A_{5} \mid B_{1}, B_{2}, B_{4}, B_{5}, B_{7}, B_{8}\right\}, \\
& \left\{A_{1}, A_{3}, A_{4}, A_{5} \mid B_{2}, B_{3}, B_{5}, B_{6}, B_{8}, B_{9}\right\},\left\{A_{1}, A_{3}, A_{4}, A_{5} \mid B_{1}, B_{3}, B_{4}, B_{6}, B_{7}, B_{9}\right\},
\end{aligned}
$$

where $\left\{A_{i_{1}}, A_{i_{2}}, A_{i_{3}}, A_{i_{4}} \mid B_{j_{1}}, B_{j_{2}}, B_{j_{3}}, B_{j_{4}}, B_{j_{5}}, B_{j_{6}}\right\}$ denotes a block such that $A_{i_{1}}, A_{i_{2}}, A_{i_{3}}, A_{i_{4}}$ are whole plot treatments and $B_{j_{1}}, B_{j_{2}}, B_{j_{3}}, B_{j_{4}}, B_{j_{5}}$, $B_{j_{6}}$ are subplot treatments in each whole plot. We note that if the ISPD is constructed by the usual Kronecker product of the incidence matrices, then the number of blocks becomes 36. Generally, the number of blocks of an ISPD constructed by the Kronecker product is $t$ times larger than those of an ISPD constructed by the semi-Kronecker product.

\section{Stratum efficiency factors for the ISPD $\tilde{\mathcal{D}}$}

In this section, we consider the stratum efficiency factors for the ISPD $\tilde{\mathcal{D}}$ constructed by the semi-Kronecker product of two $\alpha$-resolvable designs. To find the stratum efficiency factors, we need the eigenvalues of the stratum information matrices $\mathbf{A}_{1}, \mathbf{A}_{2}$ and $\mathbf{A}_{3}$ of $\tilde{\mathcal{D}}$. If the stratum information matrices $\mathbf{A}_{1}, \mathbf{A}_{2}$ and $\mathbf{A}_{3}$ have common eigenvectors, i.e., if $\tilde{\mathcal{D}}$ is generally balanced, then we can easily find the eigenvalues of the matrices. From (2),

$$
\mathbf{N}_{B i} \mathbf{N}_{B i}^{\prime} \mathbf{N}_{B j} \mathbf{N}_{B j}^{\prime}=\alpha_{2}^{2} q_{22} \mathbf{J}_{v_{2}}
$$

holds for $i, j=1,2, \ldots, t, i \neq j$. From (5) and (6), we see that the concurrence matrices $\mathbf{N}_{1} \mathbf{N}_{1}^{\prime}$ and $\mathbf{N}_{2} \mathbf{N}_{2}^{\prime}$ are commutative, which implies, by (1), that the stratum information matrices $\mathbf{A}_{1}, \mathbf{A}_{2}$ and $\mathbf{A}_{3}$ are mutually commutative. Therefore $\tilde{\mathcal{D}}$ is generally balanced.

We consider the eigenvectors of the concurrence matrices in the $\alpha_{1}$ $\mathrm{RD}\left(v_{1}, r_{1}, k_{1}\right)$ and the affine $\alpha_{2}-\mathrm{RD}\left(v_{2}, r_{2}, k_{2}\right)$ in order to find the common eigenvectors of $\mathbf{N}_{1} \mathbf{N}_{1}^{\prime}$ and $\mathbf{N}_{2} \mathbf{N}_{2}^{\prime}$.

Let $\theta_{j}^{(i)}$ and $\boldsymbol{x}_{i j}\left(j=0,1, \ldots, v_{1}-1\right)$ be the eigenvalues and the corresponding mutually orthonormal eigenvectors of $\mathbf{N}_{A i} \mathbf{N}_{A i}^{\prime}$ for each $i=1,2, \ldots$, $t$. In particular, the eigenvalue of $\mathbf{N}_{A i} \mathbf{N}_{A i}^{\prime}$ corresponding to the eigenvector 
$\boldsymbol{x}_{i 0}=\left(\sqrt{v_{1}}\right)^{-1} \mathbf{1}_{v_{1}}$ is given by $\theta_{0}^{(i)}=\alpha_{1} k_{1}$ for each $i=1,2, \ldots, t$. In Table 1 , we see the eigenvalues and the eigenvectors of the concurrence matrix $\mathbf{N}_{A i} \mathbf{N}_{A i}^{\prime}$ for each $i=1,2, \ldots, t$ in the $\alpha_{1}-\operatorname{RD}\left(v_{1}, r_{1}, k_{1}\right)$. Furthermore, the

Table 1. Eigenvalues and eigenvectors of $\mathbf{N}_{A i} \mathbf{N}_{A i}^{\prime}$ in $\alpha_{1}-\mathrm{RD}\left(v_{1}, r_{1}, k_{1}\right)$

\begin{tabular}{cc}
\hline Eigenvalues & Eigenvectors \\
\hline$\alpha_{1} k_{1}$ & $\boldsymbol{x}_{i 0}=\left(\sqrt{v_{1}}\right)^{-1} \mathbf{1}_{v_{1}}$ \\
$\theta_{j}^{(i)}$ & $\boldsymbol{x}_{i j}\left(j=1,2, \ldots, v_{1}-1\right)$ \\
\hline
\end{tabular}

eigenvalues and the corresponding mutually orthonormal eigenvectors of $\mathbf{N}_{A} \mathbf{N}_{A}^{\prime}$ are denoted by $\theta_{j}$ and $\boldsymbol{d}_{j}$ for $j=0,1, \ldots, v_{1}-1$. In particular, $\theta_{0}=t \alpha_{1} k_{1}$ and $\boldsymbol{d}_{0}=\left(\sqrt{v_{1}}\right)^{-1} \mathbf{1}_{v_{1}}$.

For the incidence matrix of the affine $\alpha_{2}-\mathrm{RD}\left(v_{2}, r_{2}, k_{2}\right)$, by $(2), \mathbf{N}_{B i} \mathbf{N}_{B i}^{\prime}$ has the eigenvalues $\alpha_{2} k_{2}, k_{2}-q_{21}$ and 0 with multiplicities $1, \beta_{2}-1$ and $v_{2}-\beta_{2}$, respectively, for each $i=1,2, \ldots, t$. From (6), $\mathbf{N}_{B 1} \mathbf{N}_{B 1}^{\prime}, \mathbf{N}_{B 2} \mathbf{N}_{B 2}^{\prime}, \ldots$, $\mathbf{N}_{B t} \mathbf{N}_{B t}^{\prime}$ are mutually commutative, so these concurrence matrices have common eigenvectors. Let $\mathbf{Q}=\left(\boldsymbol{q}_{0}, \boldsymbol{q}_{1}, \ldots, \boldsymbol{q}_{\beta_{2}-1}\right)$ be an orthogonal matrix of order $\beta_{2}$ with $\boldsymbol{q}_{0}=\left(\sqrt{\beta_{2}}\right)^{-1} \mathbf{1}_{\beta_{2}}$. For the $i$ th resolution class, by (2), the mutually orthonormal eigenvectors of $\mathbf{N}_{B i} \mathbf{N}_{B i}^{\prime}$ corresponding to the eigenvalues $\alpha_{2} k_{2}$ and $k_{2}-q_{21}$ are given by

$$
\boldsymbol{z}_{i 0}=\frac{1}{\sqrt{\alpha_{2} k_{2}}} \mathbf{N}_{B i} \boldsymbol{q}_{0} \quad \text { and } \quad \boldsymbol{z}_{i p}=\frac{1}{\sqrt{k_{2}-q_{21}}} \mathbf{N}_{B i} \boldsymbol{q}_{p}
$$

for $p=1,2, \ldots, \beta_{2}-1$, respectively. In particular, $\boldsymbol{z}_{i 0}=\left(\sqrt{v_{2}}\right)^{-1} \mathbf{1}_{v_{2}}$. By (2), the eigenvectors $\boldsymbol{z}_{i p}\left(p=0,1, \ldots, \beta_{2}-1\right)$ are also the eigenvectors of the concurrence matrix $\mathbf{N}_{B h} \mathbf{N}_{B h}^{\prime}(h \neq i)$ for the other resolution class, and the eigenvalues of $\mathbf{N}_{B h} \mathbf{N}_{B h}^{\prime}$ corresponding to $\boldsymbol{z}_{i 0}$ and $\boldsymbol{z}_{i p}(p \neq 0)$ are $\alpha_{2} k_{2}$ and 0 , respectively. The mutually orthonormal common eigenvectors of $\mathbf{N}_{B 1} \mathbf{N}_{B 1}^{\prime}, \mathbf{N}_{B 2} \mathbf{N}_{B 2}^{\prime}, \ldots, \mathbf{N}_{B t} \mathbf{N}_{B t}^{\prime}$ corresponding to the eigenvalue 0 are denoted by $\boldsymbol{z}_{q}^{*}$ for $q=1,2, \ldots, v_{2}-t\left(\beta_{2}-1\right)-1$. These eigenvalues and eigenvectors are summarized in the following table:

The common eigenvectors in Table 2 are mutually orthogonal, and these eigenvectors play an important role in the succeeding argument.

Combining the eigenvectors of Table 1 and Table 2, we consider the following six sets of vectors:
(i) $\frac{1}{\sqrt{v_{1}}} \mathbf{1}_{v_{1}} \otimes \frac{1}{\sqrt{v_{2}}} \mathbf{1}_{v_{2}}$
(ii) $\boldsymbol{d}_{j} \otimes \frac{1}{\sqrt{v_{2}}} \mathbf{1}_{v_{2}}$,
(iii) $\frac{1}{\sqrt{v_{1}}} \mathbf{1}_{v_{1}} \otimes \boldsymbol{z}_{i p}$,
(iv) $\frac{1}{\sqrt{v_{1}}} \mathbf{1}_{v_{1}} \otimes \boldsymbol{z}_{q}^{*}$,
(v) $\boldsymbol{x}_{i j} \otimes \boldsymbol{z}_{i p}$,
(vi) $\boldsymbol{d}_{j} \otimes \boldsymbol{z}_{q}^{*}$, 
Table 2. Eigenvalues and common eigenvectors of $\mathbf{N}_{B i} \mathbf{N}_{B i}^{\prime}$ in affine $\alpha_{2}-\mathrm{RD}\left(v_{2}, r_{2}, k_{2}\right)$

\begin{tabular}{|c|c|c|c|c|}
\hline \multicolumn{4}{|c|}{ Eigenvalues } & \multirow{2}{*}{ Common eigenvectors } \\
\hline $\mathbf{N}_{B 1} \mathbf{N}_{B 1}^{\prime}$ & $\mathbf{N}_{B 2} \mathbf{N}_{B 2}^{\prime}$ & $\cdots$ & $\mathbf{N}_{B t} \mathbf{N}_{B t}^{\prime}$ & \\
\hline$\alpha_{2} k_{2}$ & $\alpha_{2} k_{2}$ & $\cdots$ & $\alpha_{2} k_{2}$ & $\boldsymbol{z}_{i 0}=\left(\sqrt{v_{2}}\right)^{-1} \mathbf{1}_{v_{2}}$ \\
\hline$k_{2}-q_{21}$ & 0 & $\cdots$ & 0 & $\boldsymbol{z}_{1 p}\left(p=1,2, \ldots, \beta_{2}-1\right)$ \\
\hline 0 & $k_{2}-q_{21}$ & $\cdots$ & 0 & $\boldsymbol{z}_{2 p}\left(p=1,2, \ldots, \beta_{2}-1\right)$ \\
\hline$\vdots$ & $\vdots$ & $\vdots$ & $\vdots$ & $\vdots$ \\
\hline 0 & 0 & $\cdots$ & $k_{2}-q_{21}$ & $\boldsymbol{z}_{t p}\left(p=1,2, \ldots, \beta_{2}-1\right)$ \\
\hline 0 & 0 & $\ldots$ & 0 & $\boldsymbol{z}_{q}^{*}\left(q=1,2, \ldots, v_{2}-t\left(\beta_{2}-1\right)-1\right)$ \\
\hline
\end{tabular}

for $i=1,2, \ldots, t, j=1,2, \ldots, v_{1}-1, p=1,2, \ldots, \beta_{2}-1$ and $q=1,2, \ldots$, $v_{2}-t\left(\beta_{2}-1\right)-1$. The vectors of (i)-(vi) are mutually orthonormal and the total number of vectors is $v_{1} v_{2}$. We show that the vectors of (i)-(vi) are the common eigenvectors of $\mathbf{N}_{1} \mathbf{N}_{1}^{\prime}$ and $\mathbf{N}_{2} \mathbf{N}_{2}^{\prime}$ and we find the corresponding eigenvalues of $\mathbf{N}_{1} \mathbf{N}_{1}^{\prime}$ and $\mathbf{N}_{2} \mathbf{N}_{2}^{\prime}$.

Firstly, we consider the matrix $\mathbf{N}_{1} \mathbf{N}_{1}^{\prime}$. For (i), we have, from (5), Table 1 and Table 2,

$$
\begin{aligned}
& \mathbf{N}_{1} \mathbf{N}_{1}^{\prime}\left(\frac{1}{\sqrt{v_{1}}} \mathbf{1}_{v_{1}} \otimes \frac{1}{\sqrt{v_{2}}} \mathbf{1}_{v_{2}}\right)= \\
& =\sum_{i=1}^{t}\left(\mathbf{N}_{A i} \mathbf{N}_{A i}^{\prime} \otimes \mathbf{N}_{B i} \mathbf{N}_{B i}^{\prime}\right)\left(\frac{1}{\sqrt{v_{1}}} \mathbf{1}_{v_{1}} \otimes \frac{1}{\sqrt{v_{2}}} \mathbf{1}_{v_{2}}\right)= \\
& =\sum_{i=1}^{t}\left(\mathbf{N}_{A i} \mathbf{N}_{A i}^{\prime} \frac{1}{\sqrt{v_{1}}} \mathbf{1}_{v_{1}} \otimes \mathbf{N}_{B i} \mathbf{N}_{B i}^{\prime} \frac{1}{\sqrt{v_{2}}} \mathbf{1}_{v_{2}}\right)= \\
& =\sum_{i=1}^{t}\left(\alpha_{1} k_{1} \frac{1}{\sqrt{v_{1}}} \mathbf{1}_{v_{1}} \otimes \alpha_{2} k_{2} \frac{1}{\sqrt{v_{2}}} \mathbf{1}_{v_{2}}\right)=t \alpha_{1} k_{1} \alpha_{2} k_{2}\left(\frac{1}{\sqrt{v_{1}}} \mathbf{1}_{v_{1}} \otimes \frac{1}{\sqrt{v_{2}}} \mathbf{1}_{v_{2}}\right) .
\end{aligned}
$$

The corresponding eigenvalue is $t \alpha_{1} k_{1} \alpha_{2} k_{2}$.

For (ii), we have

$$
\begin{aligned}
& \mathbf{N}_{1} \mathbf{N}_{1}^{\prime}\left(\boldsymbol{d}_{j} \otimes \frac{1}{\sqrt{v_{2}}} \mathbf{1}_{v_{2}}\right)=\sum_{i=1}^{t}\left(\mathbf{N}_{A i} \mathbf{N}_{A i}^{\prime} \boldsymbol{d}_{j} \otimes \mathbf{N}_{B i} \mathbf{N}_{B i}^{\prime} \frac{1}{\sqrt{v_{2}}} \mathbf{1}_{v_{2}}\right)= \\
& =\sum_{i=1}^{t}\left(\mathbf{N}_{A i} \mathbf{N}_{A i}^{\prime} \boldsymbol{d}_{j} \otimes \alpha_{2} k_{2} \frac{1}{\sqrt{v_{2}}} \mathbf{1}_{v_{2}}\right)=\mathbf{N}_{A} \mathbf{N}_{A}^{\prime} \boldsymbol{d}_{j} \otimes \alpha_{2} k_{2} \frac{1}{\sqrt{v_{2}}} \mathbf{1}_{v_{2}}= \\
& =\theta_{j} \alpha_{2} k_{2}\left(\boldsymbol{d}_{j} \otimes \frac{1}{\sqrt{v_{2}}} \mathbf{1}_{v_{2}}\right) .
\end{aligned}
$$

The corresponding eigenvalue is $\theta_{j} \alpha_{2} k_{2}$ for $j=1,2, \ldots, v_{1}-1$.

For (iii), we have

$$
\begin{aligned}
& \mathbf{N}_{1} \mathbf{N}_{1}^{\prime}\left(\frac{1}{\sqrt{v_{1}}} \mathbf{1}_{v_{1}} \otimes \boldsymbol{z}_{i p}\right)=\sum_{i^{\prime}=1}^{t}\left(\mathbf{N}_{A i^{\prime}} \mathbf{N}_{A i^{\prime}}^{\prime} \frac{1}{\sqrt{v_{1}}} \mathbf{1}_{v_{1}} \otimes \mathbf{N}_{B i^{\prime}} \mathbf{N}_{B i^{\prime}}^{\prime} \boldsymbol{z}_{i p}\right)= \\
& =\alpha_{1} k_{1}\left(k_{2}-q_{21}\right)\left(\frac{1}{\sqrt{v_{1}}} \mathbf{1}_{v_{1}} \otimes \boldsymbol{z}_{i p}\right) .
\end{aligned}
$$


The corresponding eigenvalue is $\alpha_{1} k_{1}\left(k_{2}-q_{21}\right)$ for $i=1,2, \ldots, t$ and $p=$ $1,2, \ldots, \beta_{2}-1$.

For (iv), we have

$$
\mathbf{N}_{1} \mathbf{N}_{1}^{\prime}\left(\frac{1}{\sqrt{v_{1}}} \mathbf{1}_{v_{1}} \otimes \boldsymbol{z}_{q}^{*}\right)=\sum_{i=1}^{t}\left(\mathbf{N}_{A i} \mathbf{N}_{A i}^{\prime} \frac{1}{\sqrt{v_{1}}} \mathbf{1}_{v_{1}} \otimes \mathbf{N}_{B i} \mathbf{N}_{B i}^{\prime} \boldsymbol{z}_{q}^{*}\right)=\mathbf{0} .
$$

The corresponding eigenvalue is zero for $q=1,2, \ldots, v_{2}-t\left(\beta_{2}-1\right)-1$. Similarly, for (vi), the eigenvalue is also zero.

For (v), we have

$$
\begin{aligned}
& \mathbf{N}_{1} \mathbf{N}_{1}^{\prime}\left(\boldsymbol{x}_{i j} \otimes \boldsymbol{z}_{i p}\right)=\sum_{i^{\prime}=1}^{t}\left(\mathbf{N}_{A i^{\prime}} \mathbf{N}_{A i^{\prime}}^{\prime} \boldsymbol{x}_{i j} \otimes \mathbf{N}_{B i^{\prime}} \mathbf{N}_{B i^{\prime}}^{\prime} \boldsymbol{z}_{i p}\right)= \\
& =\mathbf{N}_{A i} \mathbf{N}_{A i}^{\prime} \boldsymbol{x}_{i j} \otimes \mathbf{N}_{B i} \mathbf{N}_{B i}^{\prime} \boldsymbol{z}_{i p}=\theta_{j}^{(i)}\left(k_{2}-q_{21}\right)\left(\boldsymbol{x}_{i j} \otimes \boldsymbol{z}_{i p}\right) .
\end{aligned}
$$

The corresponding eigenvalue is $\theta_{j}^{(i)}\left(k_{2}-q_{21}\right)$ for $i=1,2, \ldots, t, j=1,2, \ldots$, $v_{1}-1$ and $p=1,2, \ldots, \beta_{2}-1$.

Next, we consider the matrix $\mathbf{N}_{2} \mathbf{N}_{2}^{\prime}$. For (i), we have, from (5), Table 1 and Table 2,

$$
\begin{aligned}
& \mathbf{N}_{2} \mathbf{N}_{2}^{\prime}\left(\frac{1}{\sqrt{v_{1}}} \mathbf{1}_{v_{1}} \otimes \frac{1}{\sqrt{v_{2}}} \mathbf{1}_{v_{2}}\right)=\left(\alpha_{1} \mathbf{I}_{v_{1}} \otimes \mathbf{N}_{B} \mathbf{N}_{B}^{\prime}\right)\left(\frac{1}{\sqrt{v_{1}}} \mathbf{1}_{v_{1}} \otimes \frac{1}{\sqrt{v_{2}}} \mathbf{1}_{v_{2}}\right)= \\
& =\left(\alpha_{1} \frac{1}{\sqrt{v_{1}}} \mathbf{1}_{v_{1}}\right) \otimes\left(\mathbf{N}_{B} \mathbf{N}_{B}^{\prime} \frac{1}{\sqrt{v_{2}}} \mathbf{1}_{v_{2}}\right)=t \alpha_{1} \alpha_{2} k_{2}\left(\frac{1}{\sqrt{v_{1}}} \mathbf{1}_{v_{1}} \otimes \frac{1}{\sqrt{v_{2}}} \mathbf{1}_{v_{2}}\right) .
\end{aligned}
$$

The corresponding eigenvalue is $t \alpha_{1} \alpha_{2} k_{2}$.

For (ii), we have

$$
\begin{aligned}
& \mathbf{N}_{2} \mathbf{N}_{2}^{\prime}\left(\boldsymbol{d}_{j} \otimes \frac{1}{\sqrt{v_{2}}} \mathbf{1}_{v_{2}}\right)=\left(\alpha_{1} \boldsymbol{d}_{j}\right) \otimes\left(\mathbf{N}_{B} \mathbf{N}_{B}^{\prime} \frac{1}{\sqrt{v_{2}}} \mathbf{1}_{v_{2}}\right)= \\
& =t \alpha_{1} \alpha_{2} k_{2}\left(\boldsymbol{d}_{j} \otimes \frac{1}{\sqrt{v_{2}}} \mathbf{1}_{v_{2}}\right) .
\end{aligned}
$$

The corresponding eigenvalue is $t \alpha_{1} \alpha_{2} k_{2}$ for $j=1,2, \ldots, v_{1}-1$.

For (iii), we have

$$
\begin{aligned}
& \mathbf{N}_{2} \mathbf{N}_{2}^{\prime}\left(\frac{1}{\sqrt{v_{1}}} \mathbf{1}_{v_{1}} \otimes \boldsymbol{z}_{i p}\right)=\left(\alpha_{1} \frac{1}{\sqrt{v_{1}}} \mathbf{1}_{v_{1}}\right) \otimes\left(\mathbf{N}_{B} \mathbf{N}_{B}^{\prime} \boldsymbol{z}_{i p}\right)= \\
& =\alpha_{1}\left(k_{2}-q_{21}\right)\left(\frac{1}{\sqrt{v_{1}}} \mathbf{1}_{v_{1}} \otimes \boldsymbol{z}_{i p}\right) .
\end{aligned}
$$

The corresponding eigenvalue is $\alpha_{1}\left(k_{2}-q_{21}\right)$ for $i=1,2, \ldots, t$ and $p=$ $1,2, \ldots, \beta_{2}-1$.

For (iv), we have

$$
\mathbf{N}_{2} \mathbf{N}_{2}^{\prime}\left(\frac{1}{\sqrt{v_{1}}} \mathbf{1}_{v_{1}} \otimes \boldsymbol{z}_{q}^{*}\right)=\left(\alpha_{1} \frac{1}{\sqrt{v_{1}}} \mathbf{1}_{v_{1}}\right) \otimes\left(\mathbf{N}_{B} \mathbf{N}_{B}^{\prime} \boldsymbol{z}_{q}^{*}\right)=\mathbf{0} .
$$


The corresponding eigenvalue is zero for $q=1,2, \ldots, v_{2}-t\left(\beta_{2}-1\right)-1$. Similarly, for (vi), the eigenvalue is also zero.

For (v), we have

$$
\mathbf{N}_{2} \mathbf{N}_{2}^{\prime}\left(\boldsymbol{x}_{i j} \otimes \boldsymbol{z}_{i p}\right)=\left(\alpha_{1} \boldsymbol{x}_{i j}\right) \otimes\left(\mathbf{N}_{B} \mathbf{N}_{B}^{\prime} \boldsymbol{z}_{i p}\right)=\alpha_{1}\left(k_{2}-q_{21}\right)\left(\boldsymbol{x}_{i j} \otimes \boldsymbol{z}_{i p}\right) .
$$

The corresponding eigenvalue is $\alpha_{1}\left(k_{2}-q_{21}\right)$ for $i=1,2, \ldots, t, j=1,2, \ldots, v_{1}-$ 1 and $p=1,2, \ldots, \beta_{2}-1$. The corresponding eigenvalues of $\mathbf{N}_{1} \mathbf{N}_{1}^{\prime}$ and $\mathbf{N}_{2} \mathbf{N}_{2}^{\prime}$ are summarized in the table below:

Table 3. Eigenvalues and common eigenvectors of $\mathbf{N}_{1} \mathbf{N}_{1}^{\prime}$ and $\mathbf{N}_{2} \mathbf{N}_{2}^{\prime}$

\begin{tabular}{ccc}
\hline \multicolumn{2}{c}{ Eigenvalues } & \multirow{2}{*}{ Common eigenvectors } \\
\hline $\mathbf{N}_{1} \mathbf{N}_{1}^{\prime}$ & $\mathbf{N}_{2} \mathbf{N}_{2}^{\prime}$ & (i) \\
$t \alpha_{1} k_{1} \alpha_{2} k_{2}$ & $t \alpha_{1} \alpha_{2} k_{2}$ & (ii) \\
$\theta_{j} \alpha_{2} k_{2}$ & $t \alpha_{1} \alpha_{2} k_{2}$ & (iii) \\
$\alpha_{1} k_{1}\left(k_{2}-q_{21}\right)$ & $\alpha_{1}\left(k_{2}-q_{21}\right)$ & (v) \\
$\theta_{j}^{(i)}\left(k_{2}-q_{21}\right)$ & $\alpha_{1}\left(k_{2}-q_{21}\right)$ & (iv),(vi) \\
0 & 0 & \\
\hline
\end{tabular}

Here $i=1,2, \ldots, t$ and $j=1,2, \ldots, v_{1}-1$.

The vectors of (i)-(vi) are also the common eigenvectors of the stratum information matrices $\mathbf{A}_{1}, \mathbf{A}_{2}$ and $\mathbf{A}_{3}$. Using (1) and Table 3, we give the stratum efficiency factors for $\tilde{\mathcal{D}}$ in the following table:

Table 4. Stratum efficiency factors for the ISPD $\tilde{\mathcal{D}}$

\begin{tabular}{ccccc}
\hline $\begin{array}{c}\text { Type of } \\
\text { contrasts }\end{array}$ & No. & I & II & III \\
\hline$A$ & $v_{1}-1$ & $\xi_{j}$ & $1-\xi_{j}$ & 0 \\
$B$ & $t\left(\beta_{2}-1\right)$ & $\omega$ & 0 & $1-\omega$ \\
& $v_{2}-t\left(\beta_{2}-1\right)-1$ & 0 & 0 & 1 \\
$A \times B$ & $t\left(v_{1}-1\right)\left(\beta_{2}-1\right)$ & $t \omega \xi_{j}^{(i)}$ & $\omega\left(1-t \xi_{j}^{(i)}\right)$ & $1-\omega$ \\
& $\left(v_{1}-1\right)\left\{v_{2}-t\left(\beta_{2}-1\right)-1\right\}$ & 0 & 0 & 1 \\
\hline
\end{tabular}

Here $\xi_{j}=\theta_{j} /\left(t \alpha_{1} k_{1}\right), \xi_{j}^{(i)}=\theta_{j}^{(i)} /\left(t \alpha_{1} k_{1}\right)$ for $i=1,2, \ldots, t$ and $j=$ $1,2, \ldots, v_{1}-1, \omega=\left(k_{2}-q_{21}\right) /\left(t \alpha_{2} k_{2}\right), A$ and $B$ denote the basic contrasts among the main effects of whole plot and subplot treatments and $A \times B$ denotes the basic contrasts among the interaction effects. The eigenvectors of (ii), (iii)-(iv) and (v)-(vi) define the basic contrasts $A, B$ and $A \times B$, respectively. We use Table 4 to improve the estimators for the basic contrasts of the treatment effects combining the estimators obtained from 
the strata I, II and III. This procedure was proposed by Nelder (1965a, 1965b) and Houtman and Speed (1983). In particular, we see that some basic contrasts of $B$ and $A \times B$ are estimable with full efficiency in stratum III. For a special case where the $\alpha$-resolvable design is a resolvable design for whole plot treatments and the affine $\alpha$-resolvable design is a square lattice design for subplot treatments, the stratum efficiency factors are given in Kuriki and Nakajima (2007).

Example 2. For the ISPD $\tilde{\mathcal{D}}$ given in Example 1, $\omega=1 / 8, \theta_{1}=4, \theta_{2}=$ $\theta_{3}=2, \theta_{4}=\theta_{5}=0, \theta_{1}^{(i)}=\theta_{2}^{(i)}=2$ and $\theta_{3}^{(i)}=\theta_{4}^{(i)}=\theta_{5}^{(i)}=0$ for $i=1,2$. Thus, using Table 4 , the stratum efficiency factors can be calculated as in the following table:

\begin{tabular}{ccccc}
\hline Type of contrasts & No. & I & II & III \\
\hline$A$ & 1 & $1 / 4$ & $3 / 4$ & 0 \\
& 2 & $1 / 8$ & $7 / 8$ & 0 \\
$B$ & 2 & 0 & 1 & 0 \\
& 4 & $1 / 8$ & 0 & $7 / 8$ \\
$A \times B$ & 4 & 0 & 0 & 1 \\
& 8 & $1 / 32$ & $3 / 32$ & $7 / 8$ \\
& 12 & 0 & $1 / 8$ & $7 / 8$ \\
& 20 & 0 & 0 & 1 \\
\hline
\end{tabular}

\section{Applications}

The paper deals with a few aspects of optimal (statistical and practical) properties of experimental designs. One of the aspects relates to the proper use of the available structure of experimental units. This is very important in agricultural field experiments. The units (field plots) are natural and there is no way of changing them to a structure appriopriate to a theoretical (master) optimal design. The split-plot design, considered here, is one of the most utilized designs in agricultural field experiments (see, for example, Gomez and Gomez, 1984, Little and Hills, 1978). This results from statistical as well as practical considerations (ease of mechanically carrying out the experiment). Incomplete non-orthogonal designs (as considered here) have a much wider scope for optimal use in practice.

The second aspect concerns statistical properties of designs. Statistical properties depend on a linear model of observations. In this work we examine statistical properties of designs under a randomization-derived linear model of observations (random block effects describing the structure of units) with treatment (combination) effects being fixed (cf. Caliński and Kageyama, 2000, 2003, Hinkelmann and Kempthorne, 2005, 2007, Hinkelmann, 2012). The experimenter usually makes a 
ranking of factors as well as linear functions of level effects (contrasts) with respect to the scientific interest and aim of the experiment. Then the property called general balance plays a crucial role (see, for example, Mejza, 1992, Bailey, 1994) in satisfying the experimenter's wishes as regards the estimability and efficiency of the treatment contrasts. In the generally balanced design we know the efficiencies of all estimable treatment effect functions. Hence this property is examined here in the detail. General balance aids interpretation; the design which is generally balanced with respect to meaningful contrasts may be superior to a technically optimal design. Another problem concerns choosing an incomplete design that fits the structure of experimental units, is optimal for the most interesting treatment effect functions, and is not too expensive (it utilizes as small as possible a number of units of proper size). In the worst case we can use any incomplete design. Then it is difficult to describe the statistical properties of the proposed design.

Additionally, we seek methods enabling the generation of new incomplete splitplot designs by using some known incomplete block designs. The use of resolvable designs and affine $\alpha$-resolvable designs for the construction of new designs was not a random choice. These designs are often used in series of experiments over many environments in variety testing (see, for example, Caliński et al. 2005, 2009a, 2009b, 2015). The Kronecker product of component incomplete block designs is often used for constructing new designs with split units. The final design possesses optimal properties, but it utilizes many experimental units (high cost of the experiment). To overcome this problem (the size of the experiment) we have proposed the use of the semi-Kronecker product as defined in section 2, instead of the ordinary Kronecker product. The final design is much smaller and also possesses desirable statistical properties. Moreover, using the semi-Kronecker product to generate new designs leads to designs that use a much smaller number of units (see section 2).

\section{Acknowledgements}

The authors would like to thank to JSPS and PAN (Polish Academy of Sciences) for giving them the opportunity to carry out this joint research in Japan and in Poland. The research was partially supported by JSPS Grant-in-Aid for Scientific Research (B) 15H03636 and JSPS Grant-in-Aid for Scientific Research (C) 16K00053.

\section{REFERENCES}

Bailey R.A. (1994): General balance: artificial theory or practical relevance?. In "Proceedings of the International Conference on Linear Statistical Inference LINSTAT '93"' (T. Caliński and R. Kala, eds.), Kluwer, Dordrecht: 171-184. Springer Netherlands.

Bailey R.A., Monod H., Morgan J.P. (1995): Construction and optimality of affineresolvable designs. Biometrika 82(1): 187-200. 
Bhargava R.P., Shah K.R. (1975): Analysis of some mixed models for block and split-plot designs. Annals of the Institute of Statistical Mathematics 27: 365375 .

Caliński T., Czajka S., Kaczmarek Z., Krajewski P., Pilarczyk W. (2005): Analyzing multi-environment variety trials using randomization-derived mixed models. Biometrics 61(2): 448-455.

Caliński T., Czajka S., Kaczmarek Z., Krajewski P., Pilarczyk W. (2009a): Analyzing the genotype-by-environment interactions under a randomization-derived mixed model. Journal of Agricultural, Biological, and Environmental Statistics 14(2): 224-241.

Caliński T., Czajka S., Pilarczyk W. (2009b): On the application of affine resolvable designs to variety trials. Journal of Statistics and Applications 4(2-3): 201224 .

Caliński T., Czajka S., Kaczmarek Z., Krajewski P., Pilarczyk W., Siatkowski I., Siatkowski M. (2015): On a mixed model analysis of multi-environment variety trials: a reconsideration of the one-stage and the two-stage models and analyses. Statistical Papers: 1-33. DOI: 10.1007/s00362-015-0706-y.

Caliński T., Kageyama S. (2000): Block Designs: A Randomization Approach, Volume I: Analysis. Lecture Notes in Statistics 150, New York, Springer.

Caliński T., Kageyama S. (2003): Block Designs: A Randomization Approach, Volume II: Design. Lecture Notes in Statistics 170, New York, Springer.

Gomez K.A., Gomez A.A. (1984): Statistical procedures for agricultural research, 2nd edition. New York, John Wiley \& Sons.

Houtman A.M., Speed T.P. (1983): Balance in designed experiments with orthogonal block structure. The Annals of Statistics 11: 1069-1085.

Kadowaki S., Kageyama S. (2009): Existence of affine $\alpha$-resolvable PBIB designs with some constructions. Hiroshima Mathematical Journal 39: 293-326.

Kageyama S. (1973): On $\mu$-resolvable and affine $\mu$-resolvable balanced incomplete block designs. The Annals of Statistics 1: 195-203.

Hinkelmann K., Kempthorne O. (2007): Design and Analysis of Experiments, Introduction to Experimental Design (Volume 1), 2nd edition. Hoboken, John Wiley \& Sons.

Hinkelmann K., Kempthorne O. (2005): Design and Analysis of Experiments, Advanced Experimental Design (Volume 2). Hoboken, John Wiley \& Sons.

Hinkelmann K. eds. (2012): Design and Analysis of Experiments, Special Designs and Applications (Volume 3). Hoboken, John Wiley \& Sons.

Little M.T., Hills F.J. (1978): Agricultural Experimentation: Design and Analysis. New York, John Wiley \& Sons. 
Khatri C.G., Rao C.R. (1968): Solutions to some functional equations and their applications to characterization of probability distributions. Sankhyā A 30: $167-180$.

Kuriki S., Nakajima K. (2007): Square lattice designs in incomplete split-plot designs. Journal of Statistical Theory and Practice 1(3-4): 417-426.

Mejza S. (1992). On some aspects of general balance in designed experiments. Statistica 52(2): 263-278.

Mejza I., Kuriki S., Mejza S. (2001): Balanced square lattice designs in split-block designs. Colloquium Biometricum 31: 97-103.

Mejza I., Mejza S. (1984): Incomplete split plot designs. Statistics \& Probability Letters 2: 327-332.

Mejza I., Mejza S. (1996): Incomplete split-plot designs generated by GDP$\operatorname{BIBD}(2)$. Calcutta Statistical Association Bulletin 46: 117-127.

Mejza S. (1987): Experiments in incomplete split-plot designs. In Pukkila, T. and Puntanen, S. (Eds.), Proceeding of Second International Tampere Conference in Statistics. University of Tampere, 575-584.

Nelder J.A. (1965a): The analysis of randomized experiments with orthogonal block structure. I. Block structure and the null analysis of variance. Proceedings of the Royal Society. London, A 283: 147-162.

Nelder J.A. (1965b): The analysis of randomized experiments with orthogonal block structure. II. Treatment structure and the general analysis of variance. Proceedings of the Royal Society. London, A 283: 163-178.

Ozawa K., Kuriki S. (2006): Incomplete split-plot designs generated from $\alpha$ resolvable designs. Statistics \& Probability Letters 76: 1245-1254.

Ozawa K., Mejza S., Jimbo M., Mejza I., Kuriki S. (2004): Incomplete split-plot designs generated by some resolvable balanced designs. Statistics \& Probability Letters 68: 9-15.

Pearce S.C., Caliński T., Marshall T.F. de C. (1974): The basic contrasts of an experimental design with special reference to the analysis of data. Biometrika 61: 449-460.

Rees H.D. (1969): The analysis of variance of some non-orthogonal designs with split-plot. Biometrika 56: 43-54.

Robinson J. (1967a): Incomplete split-plot designs. Biometrics 21: 793-802.

Robinson J. (1967b): Blocking in incomplete split-plot designs. Biometrika 57: $347-350$. 